crassisuculis, infra l 1 mm longis. Fiores flavo-virentes, glaberrimi, fere $2 \mathrm{~mm}$ longi; 5-meri: sepalis basi ad $1 /$ longit. connatis, ex ovato acutis, margine minute furfuraceis, haud insignius pictis. Petala breviter connata, oblonga, apice bene rotundata, paucipunctata, intus dense papillosa. Antherae floris masculi petalis infra medium affixa, ellipticae, rotundatae, haud insignius pictae. Ovarium satis reductum, glaberrimum, conicum; stigmate minuto, capitellato.

Paraguay, bei Caaguazu in sumpfigen Gebüschen (Hassler no. 9366). - Affinis Rapaneae ovalifoliae Mez.

\title{
IV. Theophrastaceac.
}

205. Clavija gracilis Mez, spec. nov.

Ramuli tortuosi. glabri. Folia petiolis subaequa 3 iter ad $20 \mathrm{~mm}$ longis stipitata, optime elliptica nec ullo modo obovata, basi anguste longiusculeque apice late breviterque acuminata, integerrima, ad $0,2 \mathrm{~m}$ longa et $70 \mathrm{~mm}$ lata, submembranacea, glabra. utrinque optime prominuloreticulata, sub lente tenuiter sed densiuscule lineolata. Inflorescentiae pauci- (ad 20-) florae, laxe racemosae, tenuissimae, glaberrimae, foliis permulto breviores, ad $60 \mathrm{~mm}$ longae; pedicellis tenuibus usque ad $2 \mathrm{~mm}$ longis bracteas minutas, paullo lepidotas, anguste triangulares basi gerentibus. Flores minuti ad $3 \mathrm{~mm}$ diam. metientes; sepalis late ovetis, apice Jatissime acutiusculis, margine fimbriatim cren nlatis. Petala floris masculi tantum cogniti fere medium usque connata lobis 4 suborbicularibus, optime rotundatis, margine prope apicem minutissime crenulatis. Staminodia parva, globulosa, annulo carnoso glandulis epipetalèis minoribus aucto conjuncta. Stamina e fauce longe exserta tota in columnam coalita. Ovarium flori masculo deest.

Brasilien, Hylaea, am Juruà (C'le no. $57 \mathrm{~b}$ ).

\section{R. Schlechter, Studium zur Klärung der Gattung Rodriguezia Ruiz et Pav.}

In der Gatîng Rodrizuezia, wie sie heute von verschiedenen Autoren aufgefasst wird, sind ohne Zwe:fel eine Re:he recht heterogener Elemente enthalten, die wenig mit dem Typus, auf welchen hin das Genus von Ruiz and Pavon jm Jahre 1794 begründet wurte, zu tun haben.

In der Bearbeitung der Orchidaceen für die ,Flora Brasiliensis", in der die Gattung ganz offenbar ihr Entwicklungszentrum hat, finden wir bei Cogniaux drei Sektionen unterschieden, dic in folgender Weise im Bestimmungsschlüssel getrennt werden:

Folia complanata

Sepala lateralia alte connata. Labeilum indivisum. Columna libera vel basi cum columna vix connata. 
Sepala lateralja usque ad basin libera. Labellum profunde trilobatum. Colımna usque ad apicem cum labello connata . . . . . II Rodrigueziopsis Folia teretia III Capanemia

Nicht angegeben ist dabej, dass bei der ersten Gruppe das Labellum unten am Grunde stets einen fleischigen nach unten oder hint en gestreckten orler nach vorn gebogenen Auswuchs oder Sporn trägt.

Diese erste Gruppe enthält num bei Cogniaux nur solche Arten. die nach der festeren Umgrenzung der Gattung durch-Reichenbach fil. in der Botan.Ztg. X (1852)p. 770 auch wirklich zu Rodriguezia zu rechnen sind. Anders steht es aber mit den beiden anderen Sektionen.

Capanemia habe ich bereits 1914 ${ }^{1}$ ) wiederhergestellt und 1918 in Feldes Rep. XV.'p. 148 eine Aufzählung der acht hierher zu rechnenden Arten gegeben. Ein Teil von diesen war von C'ogniaux zu Rodriguezia gestellt worden. näıulich die grösseren Arten, C. uliginasa Rodr. und $C$. Juergensiana (Krïz.l) Schltr., die ïbrigen, kleineren waren zu Quekettia verwiesen worden, mit der sie nicht viel mehr als dic meist pfriemlichen Blätter gemein haben.

Betcachten wir mun Rodrigueziopsis niher, so ist eigentljch auffaliend, da's Cogniaux die bejlen hierher gerechneten Art, die von Barbosa Rodriguez als solche beschrieben worten waren. bei Rodrïgutzin belassen hat. Sic haben, von aussen gesehen, allerdings eine ähnliche Blütenform, doch schon ihre Tracht ist rerschieden, sodann sind die dïnnen Blütenschäfte mit wenigen Blüten recht abweichend. In den Blüten findeu sich Merkmale, diedoch sehr entschicden gegen die Zugchörigkeit dieser Pflanzen z.u Rodriguezia sprechen. Die zu cinem kahnförmigen Gebildeverwachsenen Sepalen scheinen mir doch, nachlem nun so vièe verschiedene Typen bekannt geworden sind, ein wichtiges Gattungsmorkmal von Rodriguezia zu sein. Sic sind bei den beiden Àrten der Sektion Rodrigueziopsis dagegen frei. Wichtig ist ferner, dass bei Rodriguezia die aus schmaiem Grunde nach vorn verbreitete, gewöhnlich ungeteille oder rom eingeschnitlene Lippe frei von der Säule ist und oberseits nur e:n bis mehrere dünne schmale Kicie trägt, bei der Sektion Rcdrigutziopsis dagegen mit den' Säulenränlern fest verwachsen ist und dann in eine sehr deutlich dreilappige Platte verbreitert ist, am Grunde zwei längliche Sichwieie trägt und den spornartigen Auswuchs unten au Cirunde. der bej Rodriguczia von den seitlichen Sepalen unschlossen wird, nicht besitzt. Dazu kommt. dass die Säule bei den bejden Cognia u x schen Sektionen recht verschicden ist.

Fassen wir diese Unterschiedo zusammen, so müssen wir ganz entschieden zu dem Schlusse kommen, dass wir hier nicht zwei Sektionen, sondern zwei recht gut getrennte Gattungen vor uns haben, die nicht einmal sehr nahe miteinander verwandt sind. Die Sektion Rodrigueziopsis Cogniauxs inuss also zum Range einer Gattung erhoben werden. auf

1) Cfr. Schltr., die Orch. (1914) p. 458 
we'che ich den von Cogniaux gebildeten Sektionsnamen übertrage. Die neue Gattung charakterisiere ich folgendermassen.

Rodrigueziopsis Schltr. nov. gen.

Sepala oblonga, obtusa, connıventia, basin versus leviter angustata, lateralia obliqua. Petala sepaljs similia, obliqua, paululo breviora. Labellum columnae marginibus alte adnatum, e medio trilobum, labis lateralibus erectis, obtusis, intermedio decurvulo, oblongo vel suborbiculari, antice emarginato vel exciso, quam lat erales bene majore, carinis 2 obtusis apicem versus sensim paululo dilatatis, subparallelis e basi labelli nsque in basin lobi intermedij decurrentibus ornatum. Columna mediocris, erecta, marginibus labello alt e adnata, semiteres, apice margine utrinque in auriculam triangulam obtusa $m$ erectam exeuns; clinandrio postice levit er descendente; rostello brevi, erecto, triangulo, exciso. Anthera adseendens ovato-cucullata. Pollinia 2 pyriformia, stipite spathulato, dimidio superiore conspicue dilatato, polliniis fere duplo longiore, viscidio elliptico, parvulo. Stigma leviter excavatum rotundatum. Ovarium pedicellatum, glabrum, haud tortum.

Plantae gracillimac. epiphyticae; rhizomate pergracili, dichotome ramoso, vagante, perlaxe pseudobulbis obsesso, ad basin pseudobulborum raclicante; radicibus filiformibus, elongatis, gracillimis, simplicibus, glabris; pseudobulbis lateraliter valde compressis, ovatis, bifoliatis, latere utrinque vaginis $2-3$ (quarum $1-2$ foliiferis) basi protectis; foliis erectopatentibus, oblongo-lanceolatis, texiura tenuior.bus; inflorescentis suberectis, in axillis vaginarum superiarum ad basin pseudobulborum natis, folia aequantibus vel paulo superantibus, laxe pauci (1-3-) flor:s, pedunculo gracillimo (nunc setiformi), pauci raginulato; bracteis parvulis; ovario pedicellato multo breviorius; flor bus vix mediccribus, tenuibus, albis, nune labello purpureo-notatis.

Species 2 althuc notae, silvarum Brasiliensium indigenae.

Auf die Unterschiede zwischen Rodrigueziopsis und Rodriguezia bin ich schon oben ausführlicher eingegangen. Schwieriger ist es, die Verwandtschaft der neuen Gattung festzustellen. Die Struktur des Labellums und der Säule sprechen entschiedèn gegen e ne sehr nahe Verwandtschaft mit Kodriguezia, dagegen erinnert die Säule mehr an Triztuxis und C'apanemia. von denen Rorlrigueziopsis aber durch die Tracht und die mit der lippeanden Rändern verwachsene Säule sich unterscheidet. Immerhin dürtte sie zunachst am besten in deren Niahe verwiesen werden.

Die beiden Arten von Rodrigueziopsis sind:

1. R. cleutherosepala (Rodr.) Schltr. nov. comb.

Rodriguezia eleutheroscpala Rodr., Orch. Nov. II (1882), p. 240.

Brasilien: In den Urwäldern auf dem Gipfel der Serra da Prata, Estado Parana, 600 m ü. M. - Ba rbosa Rod riguez, blühend im Februar; Ypiranga. Serra do Mar. Parana. -- P. Du sen no. 3789, im Februar 1904.

Von der zwe.ten Art, $R$. microphyton (Rodr.) Schltr. ist $R$. eleutheroepala (Rodr.) Schltr. verschieden durch etwas grössere, gelblich-we sse. 
auf der Lippe und an der Säulenspitze rotgetuschte Blüt en mit jängeren Se.tenlappen und rundem, am Rande gewe!item, vorn ausgeschnitterem M.ttellappen sowie die etwas iängere, daher auch schlankere Säule. Im Wuchs ist sie auch etwas stärker als $R$. microphyton (Rodr.) Schltr.

2. R. microphyton (Rodr.) Schitr. nov, comb.

Rodriguezia microphyta Rodr., Rev. d. Eng. III (1882), p. 144 ; Orch. Nov. II (1881), p. 241.

Brasilien: In provincia Rio de Janeiro - Glaziou no. 17263; no. 17799; in silvis umbrosis humidisque ad Serra de Santa-Anna Barbosa Rodriguez; Serra dos Orgaos. - Gardener no. 667; auf Bäumen bei Theremopol:s - K. Schenck no. 2696, im Febr. 1887; .J. De Moura no. 96 ; no. 412 : auf Bäumen der Wälder bei Novo Friburgo. E. Ule no. 3473, im Januar 1898; Sao Paulo--St. Hi laire no. C 21638 ; bei Campo Grande. Sao Pauı -. Comissao Geogr. et Geor. no. 3280.

Die Unterschieile zwischen dieser Art und $k$. eleatherosepala (Rodr.) Schitr. sind schon oben besprochen worden. Ich möchte hier nur noch hinzufügen, dass bei Rodrigueziopsis microphyton (Rodr.) Schitr. die Setenlappen des Labellums stumpf und schief dre eckig sind, der Mittellappen länglich bis länglich-ciförmig, an der spitze le:cht ausgerandet.

Nachdem wir Capanemia und Rodrigueziopsis aus der Gattung Rodriguezia entfernt haben. bleiben in der, ,Flora Brasilienis" nur noch Arten übrig. we'che als echte Rodriguezien bezeichnet werdert können. Neuerdings hat aber nun Kränzlin eine Pflanze aus Matto-Grosso als Rodriguezia Lindmanii Kränzl. beschrieben, die ebenfails nicht zu Rodriguezia gehört. Da mir leider kein Material dieser Pflanze zugänglich ist, muss ich die Feststellung ihrer wahren Verwandtschafi, trotz der guten Abbildung Lindmans in den Kgl. Svensk. Vet. Akad. Handl. XINT (1911), no. 10, t. 12, fig. 7 auf spätere Zeiten verschieben, bis besseres Material der Art vorliegen wird. Bemerken möchte ich nur, dass die Pflanze $m^{\prime} r$ in ke ne der bisher beschr ebenen Gattungen hine nzupassen scheint, wenigstens sowe $t$ sich aus der allerdings recht guten Abbildung ein SchluB zichen lässt.

Es findet sich nun in Zentral-Amerika noch ein abwe:chender Typus, der bei Rodriguezia untergebracht worden ist. Ihn näher anfzuklären ist erst jetzt möglich, nachdem gutes Material vorlicgt. Fs handelt sich um die Pflanze, welche im Jahre 1895 als Rodriguezia inconspicua Kränzl. beschrieben worden ist. Schon e:n Blick auf die Tafel, welche damals von ihr im Bulletin de l'Herbier Boissier v. III. (t. 18) erschicnen ist, und die Beschreibung lassen vermuten, dass hier kaum cine Rodrigurzia vorliegen kann.

Ich hatte während der letzten zohn Jahre wichlerholt aus (Josta Rica Material erhalten, dass ich für diese Pflanze hie't, doich war ich me ner Bestimmung nicht ganz sicher, da das dargestelite Exemplar der $R$. inconspicua Kränzl. eigentlich in allen hauptsächlichsten Merkmalea abzuweichen schien. Da mir jedoch die Art der Arbeit des Herrn Profi. 
Kränzlin nur zu wohl bekannt war, vermutete ich auch hier wieder eine Karrikatur und oberflächliche Untersuchung. Mit dem Herbar Kränzlin kam dann anch das Original der $R$. inconspicua Kränzl. in den Bes.tz des Botanischen Museums in Dahlem, wo ich die Pflanze genau vergle chen konnte. Es stellte sich heraus, dass meine Vermutung die richtige war. Die Pflanze ist nicht nur ganz schlecht abgebildet, sondern auch ganz falsch beschrieben worden und ist zudem voliständig identisch mit ener anderen, we'che, ebenfalls von Kränzlin, vor vier Jahren als Rodriguezia Candelariae Kränzl. bekannt gegeben ist. Sowohl habituell wie auch in der Struktur der Blüten ist sie von Rodriguezia generisch verschieden und wohl näher mit Trizeuxis verwandt. Sie ist Typus einer neuen Gattung, welche ich hier begrïnden möchte. Auf die unterscheidenden Merkmale gehe ich dánn unten nähẹ ein.

Hybochilus Schltr. nov. gen.

Sepala conniventia, obionga, obtusa, glabra, concavula, lateralia obliqua, usque ad tertiam partem apicalem connata. Petala. oblique ovata, obtusa, glabra. quam sepala paululo breviora. Labellum sessile circutu ovato-oblongum, panduratum, ecalcaratum, glabrum, dimidjo inferiore suborbicular-ovato, concavulo, medjo feregibbo depresso, rel rorso, semiquadrato ornatum, dimidio anteriore e basi angustiore apicem versus sursum paulo dilatatum, apice truncatissimo leviter exciso subbilobuatum, totum sepala dimidio fere superans, inedio (in parte constricta) cost is 2 obscuris, parallelis donatum. Columna brevis, erecta, glabra antice apicem versus malieoliformi-producta, i. e. brachiis 2 oblique ovatotriangulis, porrectis, obtusis donata sepalis multo brevior. Anthera incumbens, ovato-cucullata, obtusa, glabra, dorso gibbo satis magno ornata . Pollinia 2 oblique oblanceolata. stipite lineari, basin versus sensin paulo angustato viscidio anguste oblongo, stipite àequilongo affixa. Ovarium pedicellatum, clavatum, glabrum.

Planta epiphytica, spithamaea usque pedalis; rhizomate valde abbreviato; radicibus filiformibus, flexuosis, parum ramosis, glabris; pseudobulbis bene approximatis, suborbicularibus vel subquadratis valde compressis, unifoliatis, vagina nunc foliacea, latere utrinque donatis; foljo crecto-patente, oblongo, corjaceo; inflorescentia erecta, ex axillis vaginarum juxta basin pseudobulborum nata, vulgo pluriramosa, graciliter pedunculata, laxe multiflora: bracteis elliptico-lanceolatis, acuminais, subpatentibus, ovario pedicellato brevioribus: floribus parvulis, glabris, extus purpurascentibus, labello albo.

Species singula adhue nota costaricensis.

D:e Gattung dürfte am besten in die.Nähe von Trizeuxis-verwiesen werden, mit der sie in der allgemeinen Struktur der Blüte ganz gut übereinstimmt, von der sie-aber durch die Tracht, den Bau des Labellums die kürzere Säule mit deutlicheren Armen und die Anthere verschieden ist. Die Pollinarien sind bei beiden Gattungen recht ähnlich. Sicher ist. dass die Pflanze nit Rodriguezia nichts zu tun hat. 
Ausser der hier za der Gattung verwiesenen Art gehört vielle cht noch Rodriguezia leochilina Rchb. f. hierher. Infolge Mange!'s an Material konnte diese Frage leider nicht endgïltig entschieden werden, trotz e ner mit giitigst übersandten Pause der Pflanze im Herb. Reichenbach, für die ich Herrn Prof. Dr. Zahlbruckner und Herrn Dr. v. Keissler auch an dieser Stelle meinen Dank aussprechen möchte.

Der Typus der Gattung ist :

Hybochilus inconspicuus (Kränzl.) Schltr. nov, comb.

Rodriguezia inconspicua Kränzl., in Bull. Herb. Boiss. IIJ (1895), p. 630 .

Rodriguezia Candelariae Kränzl.. im Engl. Jahrb. v. I.IV (1916), Be:bl. 117, p. 32.

Costa Rica: San José - A. Tonduz; an Flußufern bei San José A. C. Brade no. 39, in Februar 1908 ; Los Frailes, $1548 \mathrm{~m}$ ü. M. - A. Tonduz. no. 7887. im April 1893; auf Bäumen auf dem Candelaria-Gebirge Dr. C. Hoffinann, im Februar 1856.

Nach Angabe von Herrn Bràde sind die Bliiten aussen schmutzig karminrot mit weisser karminrot getïpfelter Lippe.

Kränzlin glaubt, dass die Pflanze mit Trichocentrum candidum Idl. identisch ist, - ich kann mich dazu nicht äussern, da ich die Lindleysche Pflanze nicht kenne. Mit seiner kurzen Beschreibung stimmt unsere Pflanze jełenfalls keineswegs in allen Punkten überein.

\section{Cyperaceae novae. V.}

Von Dr. G. Kükenthal (Koburg).

40. Cyperwe sandwicensis Kükenthal. - C. strigosus Hillebr. FI. Haw. Isl. 467 (incl. var. B.), non L.

Rhizoma breve durum. Culmus submetralis validus acutangulus superne scabriusculus basi incrassatus. Folia culmo breviora longiorav $\theta$ 10-12 mm lata plana subtus scaberrima coriacea, vaginae longae purpurascentes. Anthela composita ampla laxa 10-12-radiata, radii firmuli ad $10 \mathrm{~cm}$ longi superne pluriramosi, rami divaricati breves alterni Bracteae 6-8 patentes inferiores anthelam longe superantes. Spiculae in spicas oblongo-ovatus $2 \mathrm{~cm}$ longas laxe dispositae divergentes bracteola brevi aristaeformi suffultae lineari-lanceolatae acutae subcompressa $\theta$ 7-9 $\mathrm{mm}$ longae $1 \mathrm{~mm}$ latae 4-8-florae. Squsmse inferiores vacuae persistentes, superiores laxe imbricatae demum patulae oblongo-lanceolatae obtusiusculae sub apice mucronulatae dorso tenuiter 7-9-nervisa viridicarinatae lateribus stramineo-rufae vel fuscae. Nux in specim. visis non evoluta. Stylus longus profunde trifidas exsertus. Stamina 3. Rhacheola tenuis angusto alata.

Sandwich-Inseln: Molokai, on higb palis 2000' (Hillebrand!), 